\title{
Study of Ego Virtues among Tribal and Non -Tribal, Male and Female College Students
}

\author{
Dr Ushakiran Agrawal \\ Research Project Sponsored By U.G.C.Bhopal (M.P.) India
}

\begin{abstract}
The paper is a part of my research project on STUDY OF EGO VIRTUES AMONG TRIBAL AND NON -TRIBAL, MALE AND FEMALE COLLEGE STUDENTS", the objective was to prepare and compare sex wise and culture wise ego virtues profiles, The sample of the present study was of college going 100 males and 100 females of tribal region (Preferably Gond tribe) Their age range was from 18 yrs to 23 yrs they were, under graduate and post graduate students. Similarly 100 male sand 100 females of non -tribal region were chosen having the same age group and same educational qualification. Ego virtues Inventory developed by $\mathrm{Dr} R$ $D$ Helode has been used. It is based upon the Erikson's personality theory reflecting psychosocial balance, consisted of 80 items, ten items for measuring each virtue, there are in all 8 virtues to be measured namely hope , will, purpose, competence, fidelity, care and wisdom, the split half (odd-even) reliability of this inventory as well as sub scale wise is as under; for hope scale it is 0.57 , for purpose scale it is 0.59 , for competence scale it is 0.79 , for fidelity scale it is 0.66 ,for love scale it is 0.82 ,for care scale it is 0.89 , for wisdom scale it is 0,88 and for the whole inventory it is 0.95.. Profiles are matching for tribal's and non tribal's on virtue, will and purpose, while they differ on hope, competence, fidelity, love, care and wisdom. Sex wise profile of boys and girls match on love and differ on hope, will. Purpose, competence, fidelity, care and wisdom, therefore counseling is needed for bringing different groups on parity.
\end{abstract}

\section{Introduction}

The term personality comes from Latin word Persona meaning mask. According to Greeks the actor wore masks to hide their identity and to enable them to represent the character they were depicting in the play . This dramatic technique was later adopted by the Romans and from them we get our modern term personality .The Romans persona meant "as one appears to others not one actually is". From this connotation of the word persona our popular idea of personality has been derived.

According to Allport (1961) personality is the dynamic organization within the individual of these psychophysical systems that determine the individuals is unique adjustment to the environment. The term dynamic points up the changing nature of personality it emphasizes that changes can occur in the quality and quantity of a person's behavior. Organization implies that personality is not made up of a number of different traits. One simply added to the other but that they are inter-related. The inter-relationship changes with some traits becoming more dominant and other less so, with changes in the child and in the environments. The psychophysical systems are the habit attitudes values beliefs, emotional states Sentiments and motives which are Psychological in nature but which have a physical basis in the person's neural glandular and general bodily states. These systems are not the product of heredity though they are based on hereditary foundations. The psychophysical systems are the motivating forces which determine what kind of adjustment the person will make.

Erikson's theoretical formulation proceeds by stages, eight in all according to Erikson's time table first four stages occur during infancy and childhood fifth stage during adolescent and the last 3 stages during the adult yrs up to and including old age. In Erikson's writings particular emphasis is placed on adolescent period because it is them that the transition between childhood and Adulthood is made what happens in this stage is of greatest significance for adult personality. Identity crisis and identity confusion are undoubtedly the most familiar of Eriksonian's concepts. Erikson has been described as an ego psychologist studying the stages of development spanning the entire life span. Each of Erikson's stages of psychosocial development are marked by a conflict for which successful resolution results in a favorable outcome for example trust vs mistrust and by important event that this conflict resolves itself around for example meaning of one's life. Favorable outcome s of each stage are sometimes known as Virtues a term used, in context of Erikson an work as it is implied to medicines meaning potencies. e.g. the virtue that would emerge from successful resolution. Erikson's research suggests that each individual must learn how to hold both extremes of each life stage challenge in tension with one another ,not rejecting one end of tension or the other. only when both extremes in a life stage are understood and accepted as both required and useful, can the optimal virtue for that stage surface. thus trust and 
mistrust must both be understood and accepted in order for realistic hope to emerge as a viable solution at the first stage. The Erikson's life stage virtues called ego virtues.

Erikson was a Neo-Freudian. He has been described as an "ego psychologist" studying the stages of development, spanning the entire lifespan. Each of Erikson's stages of psychosocial development are marked by a conflict, for which successful resolution will result in a favorable outcome, for example, trust vs. mistrust, and by an important event that this conflict resolves itself around, for example, the meaning of one's life.

Favorable outcomes of each stage are sometimes known as "virtues", a term used, in the context of Erikson a work, as it is applied to medicines, meaning "potencies." Erikson's research suggests that each individual must learn how to hold both extremes of each specific life-stage challenge in tension with one another, not rejecting one end of the tension or the other. Only when both extremes in a life-stage challenge are understood and accepted as both required and useful, can the optimal virtue for that stage surface. Thus, 'trust' and 'mistrust' must both be understood and accepted, in order for realistic 'hope' to emerge as a viable solution at the first stage. Similarly, 'integrity' and 'despair' must both be understood and embraced, in order for actionable 'wisdom' to emerge as a viable solution at the last stage.

The Erikson life-stage virtues, in the order of the stages in which they may be acquired, are:

1. Basic trust vs. basic mistrust - This stage covers the period of infancy. 0-1 year of age. - Whether or not the baby develops basic trust or basic mistrust is not merely a matter of nurture. It is multi-faceted and has strong social components. It depends on the quality of the maternal relationship. The mother carries out and reflects their inner perceptions of trustworthiness, a sense of personal meaning, etc. on the child. If successful in this, the baby develops a sense of trust which "forms the basis in the child for a sense of identity.

2. Autonomy vs. Shame - Covers early childhood - Introduces the concept of autonomy vs. shame and doubt. During this stage the child is trying to master toilet training.

3. Purpose - Initiative vs. Guilt - Preschool / 3-6 years - Does the child have the ability to or do things on their own, such as dress him or herself? If "guilty" about making his or her own choices, the child will not function well. Erikson has a positive outlook on this stage, saying that most guilt is quickly compensated by a sense of accomplishment.

4. $\quad$ Competence - Industry vs. Inferiority - School-age / 6-11. Child comparing self worth to others (such as in a classroom environment). Child can recognize major disparities in personal abilities relative to other children. Erikson places some emphasis on the teacher, who should ensure that children do not feel inferior.

5. Fidelity - Identity vs. Role Confusion - Adolescent / 12 years till 20. Questioning of self. Who am I, how do I fit in? Where am I going in life? Erikson believes that if the parents allow the child to explore, they will conclude their own identity. However, if the parents continually push him/her to conform to their views, the teen will face identity confusion.

6. Intimacy vs. isolation - This is the first stage of adult development. This development usually happens during young adulthood, which is between the ages of 20 to 24. Dating, marriage, family and friendships are important during the stage in their life. By successfully forming loving relationships with other people, individuals are able to experience love and intimacy. Those who fail to form lasting relationships may feel isolated and alone.

7. Generatively vs. stagnation is the second stage of adulthood and happens between the ages of 25-64. During this time, people are normally settled in their life and know what is important to them. A person is either making progress in their career or treading lightly in their career and unsure about if this is what they want to do for the rest of their working lives. Also during this time, a person is enjoying raising their children and participating in activities that gives them a sense of purpose. If a person is not comfortable with the way their life is progressing, they're usually regretful about the decisions and feel a sense of uselessness.

Ego integrity vs .despair. This stage affects the age group of 65 and on. During this time you have reached the last chapter in your life and retirement is approaching or has already taken place. Many people who have achieved what was important to them look back on their lives and feel great accomplishment and a sense of integrity. Conversely, those who had a difficult time during middle adulthood may look back and feel a sense of despair. As an evolving work in progress. This summary attempts to show the main points of the Erikson psychosocial crisis theory of human development. More detail follows this overview.

Franz And Kathleen (2008) studied individuation and attachment in personality development: Extending Erikson's theory; The question of whether Erikson's theory of psychosocial development is a complete and coherent view of development in males and females is considered. After a thorough review of Erikson's views on the role of sex in psychosocial development, the authors suggest that an important element is neglected in Erikson's account of personality development in both sexes. That is, due to his focus on issues of identity, Erikson does not account fully for the development of intimacy or other expressions of interpersonal attachment. The authors conclude that the major shortcoming of Erikson's theory is not, as some feminists have argued, that it is a male theory but that it fails to account adequately for the 
processes of interpersonal attachment that are essential to the development of both males and females. Preliminary elements of a two-path model of development are proposed.

\section{OBJECTIVE}

One of objective of this research project is as follows:

1. To prepare and compare sex wise and culture wise ego virtues profiles

\section{Methodology}

Sample:

The sample of the present study was of college going 100 males and 100 females of tribal region (Preferably gond tribe) Their age range was from $18 \mathrm{yrs}$ to $23 \mathrm{yrs}$ they were, under graduate and post graduate students. Similarly 100 male sand 100 females of non -tribal region were chosen having the same age group and same educational qualification.

Tools:

Ego virtues Inventory developed by Dr R D Helode has been used. It is based upon the Erikson's personality theory reflecting psychosocial balance, consisted of 80 items, ten items for measuring each virtue, there are in all 8 virtues to be measured namely hope, will, purpose, competence, fidelity, care and wisdom, the split half ( odd-even) reliability of this inventory as well as sub scale wise is as under; for hope scale it is 0.57 , for purpose scale it is 0.59 , for competence scale it is 0.79 , for fidelity scale it is 0.66 ,for love scale it is 0.82 , for care scale it is 0.89 , for wisdom scale it is 0,88 and for the whole inventory it is 0.95 ..

So far as validity is concerned it can be said that this inventory is standing with construct validity as the inter correlations among the scores of eight virtues are statistically insignificant, this observation indicates that each ego virtue comes out separately due to successful resolution of psychosocial crisis of each developmental stage as assumed by Eriksons theory

\section{Procedure:}

Data was collected in small group of five .After establishing rapport with them, then Ego virtues Inventory was administered. And scoring was done.

\section{Data Analysis;}

Mean was computed to prepare sex wise and culture wise, ego virtue profiles.

III. Result and Discussion:

First objective was to make sex wise and culture wise ego virtue profiles were prepared and the same are presented below

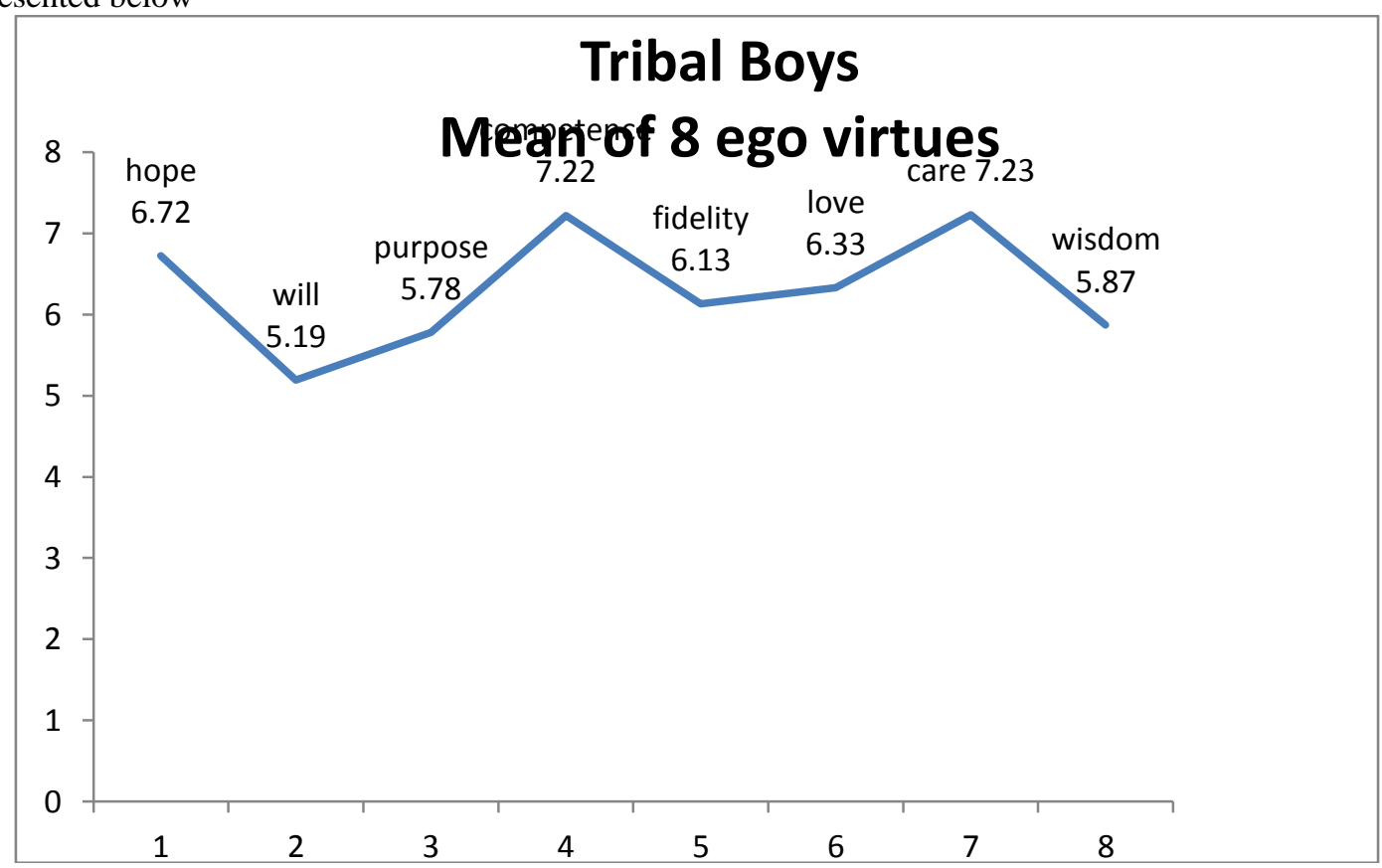

Among tribal boys mean are highest 7.22 in competence ego virtue and lowest on will ego virtue 5.19 


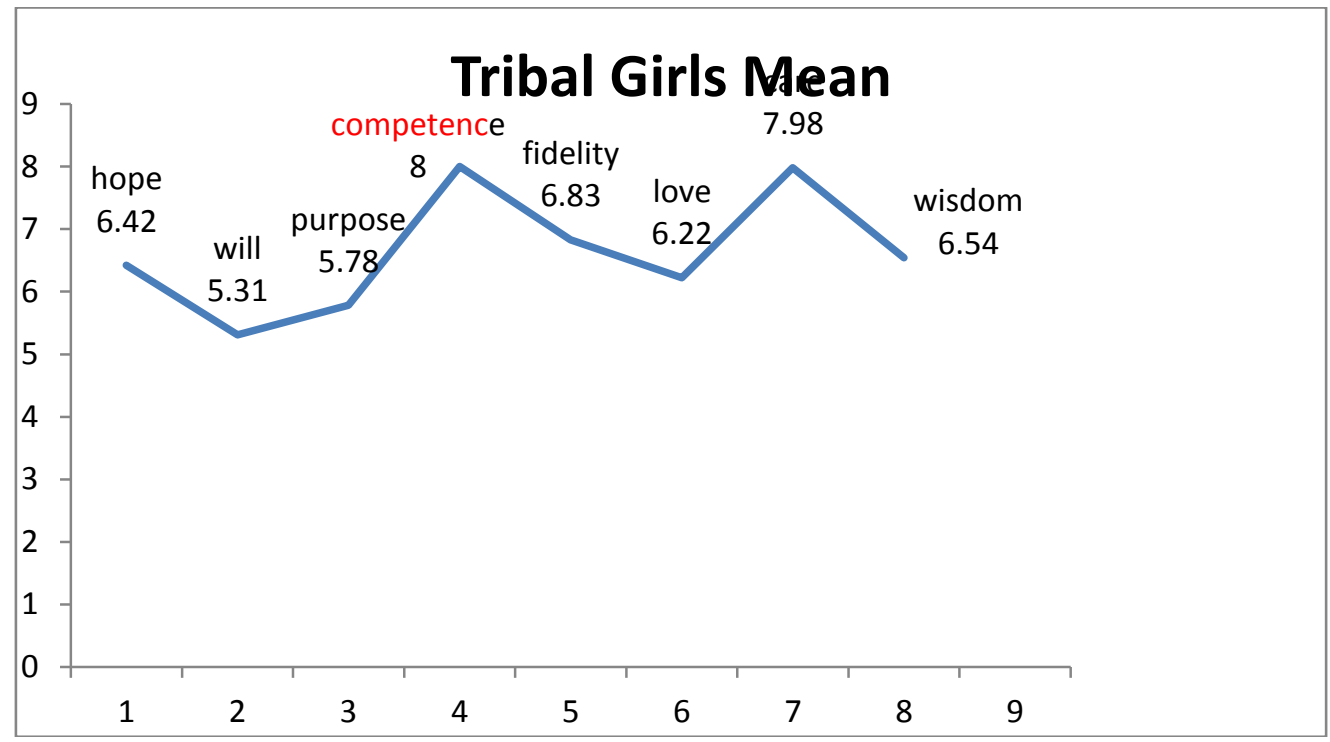

Among tribal girls mean is highest i.e. 8 on competence ego virtue and is lowest on will virtue i.e. 5.31

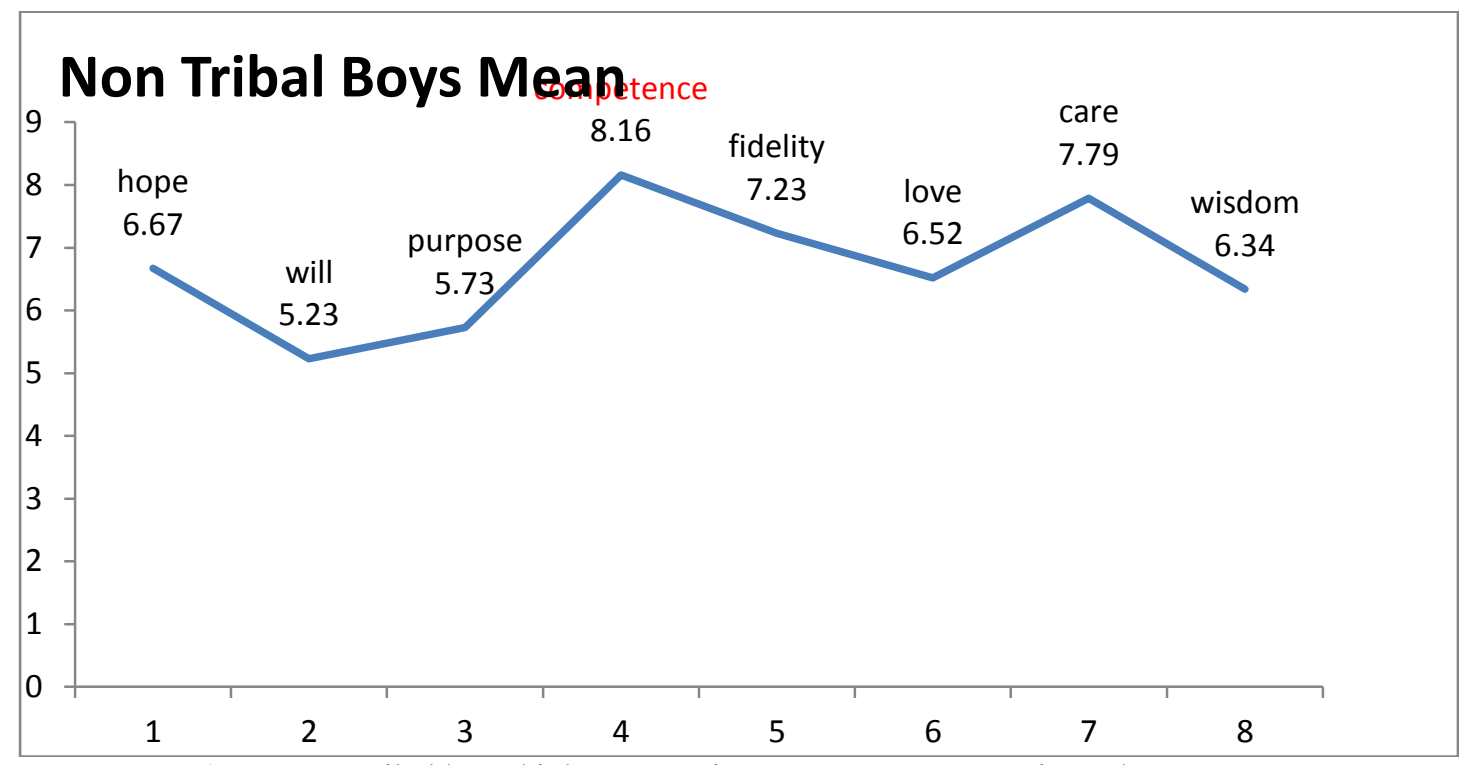

Among nontribal boys highest mean is on competence ego virtue than on care

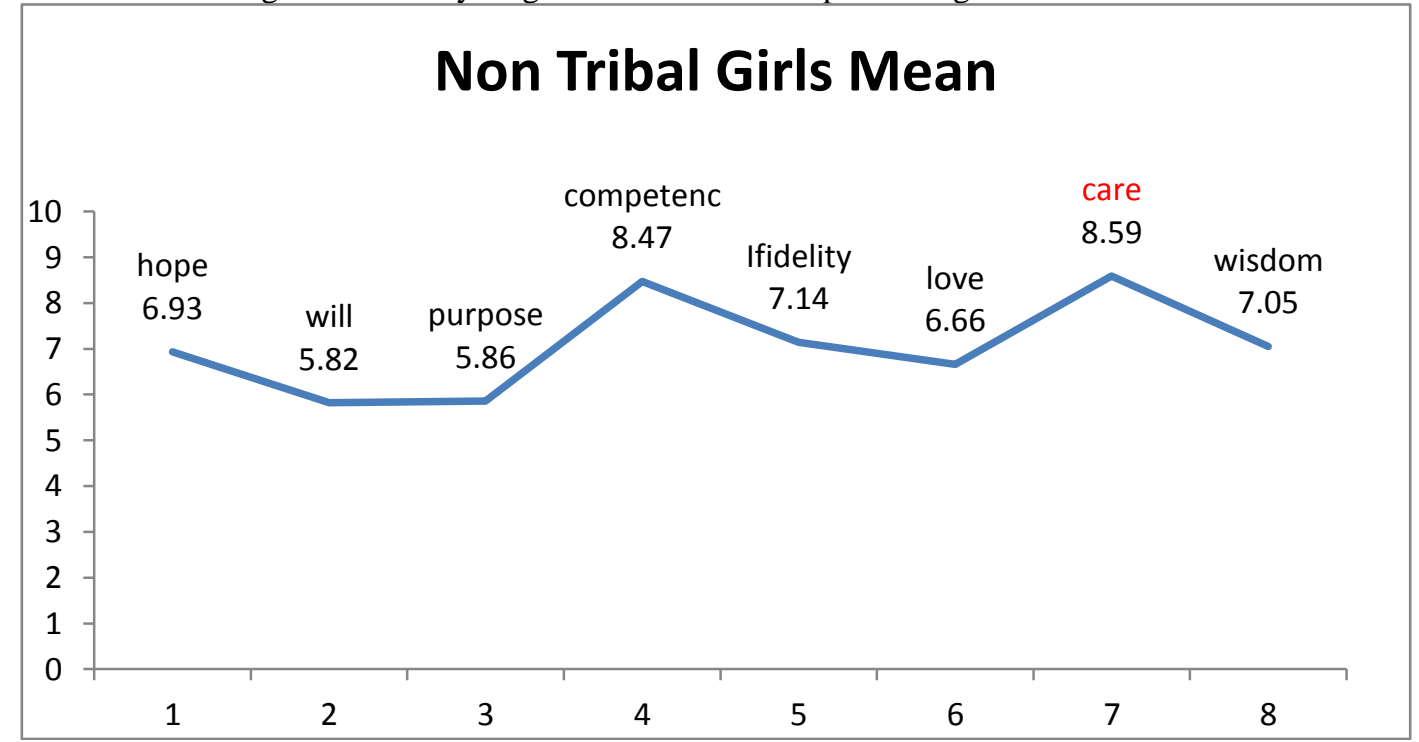

Among non tribal girls highest mean is on care ego virtue i.e.8.59. 


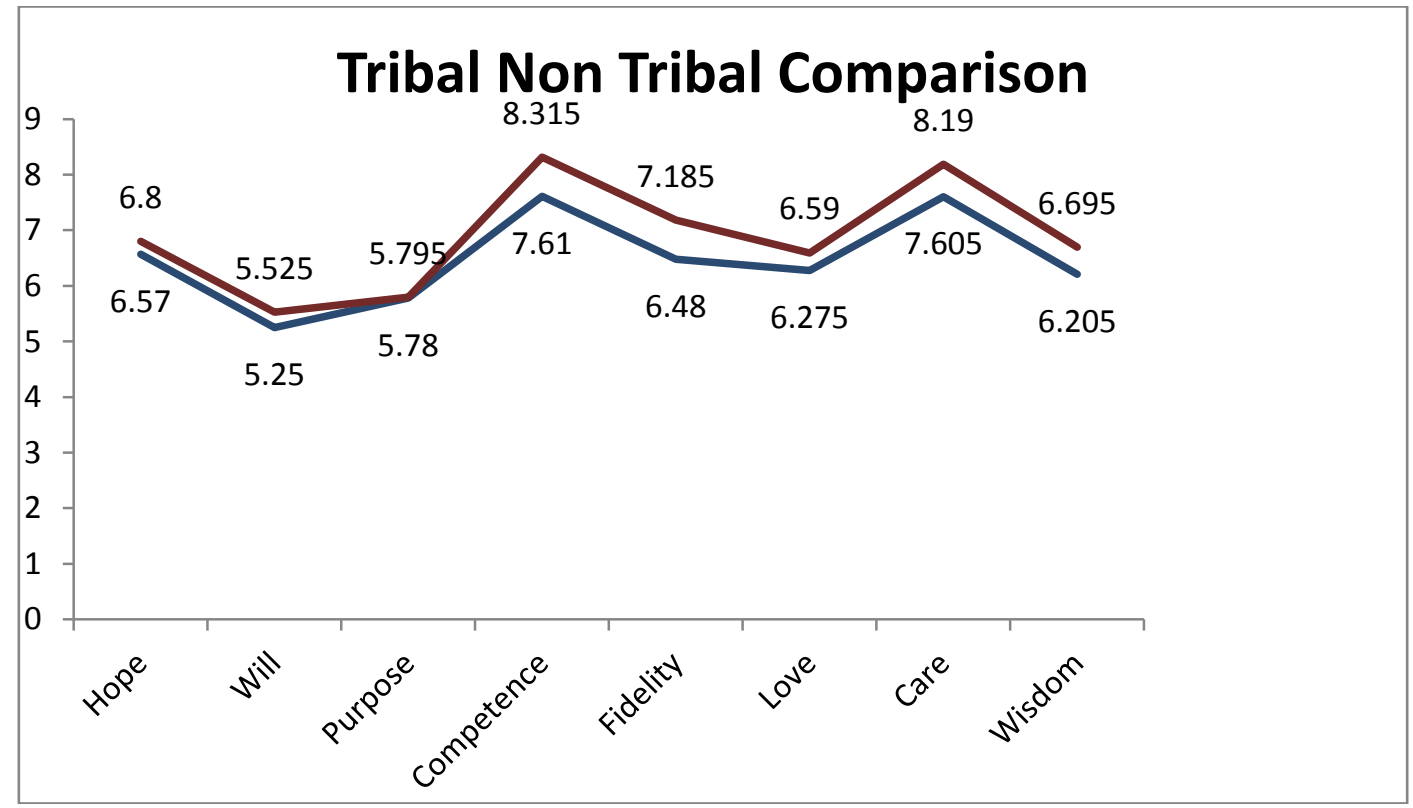

Culture wise comparison of profiles of ego virtues.

\section{Ego virtue profile according to sex}

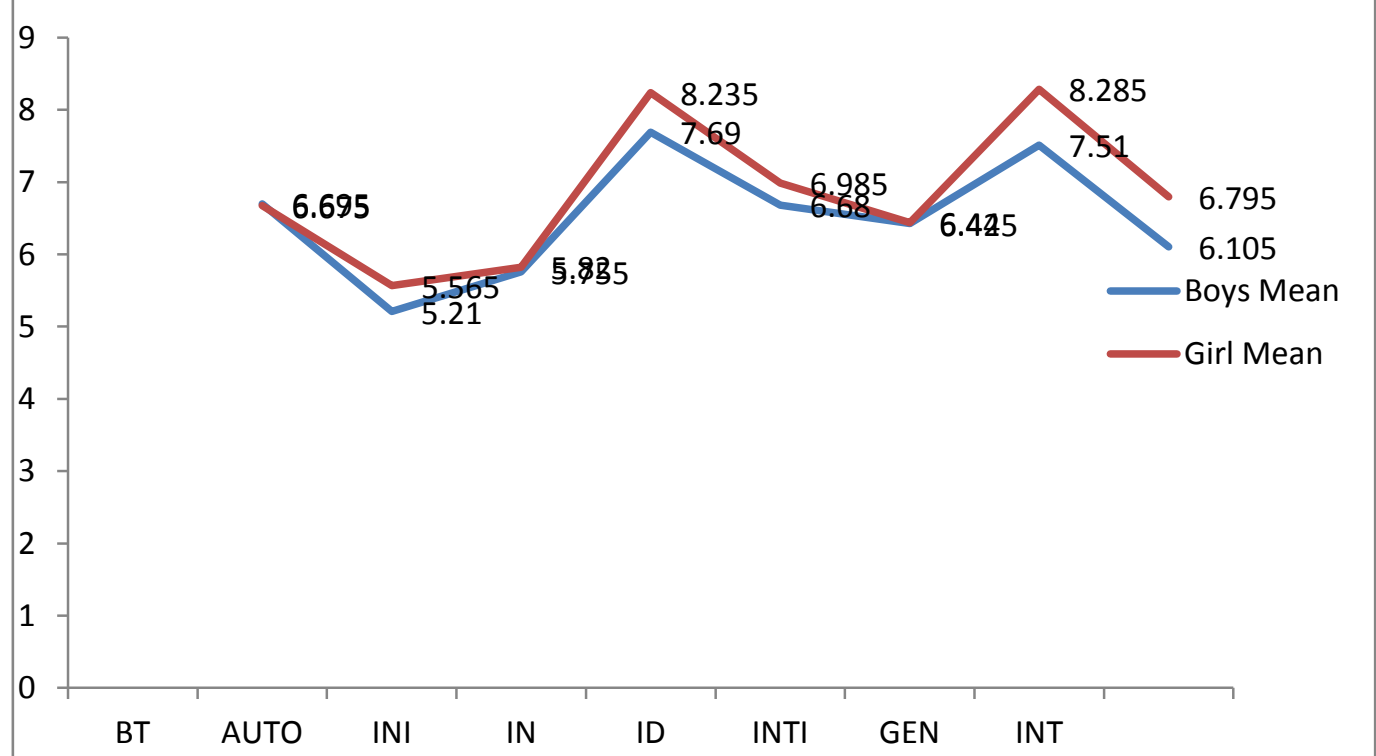

Sex wise Ego virtue profiles

Sex wise interpretation of ego virtues

Hope ; the mean for virtue hope for boys and girls 6.69 and 5.67 respectively, it was more for girls, the difference was due to variation in rearing up practices of boys and girls and child's relationship with mother .

Will ; in this virtue the mean of boys and girls were 5.21 and 5.56 respectively, was more for girls than boys, the difference may be due to the vitality and endurance of sex hormone among girls.

Purpose; the mean among boys and girls in this virtue was 5.75 and 5.82 i.e. more among girls as compared to boys. Girls have a higher mean because girl has a significant relationship with family hence develop purpose.

Competence ; the mean of this virtue of boys and girls was 7.69 and 8.23 , i.e. higher for girls and lower for boys girls were higher on this virtue must have received more encouragement and commendation by parents and teachers hence they developed a feeling of competence more as compared to boys .

Fidelity; means of boys and girls were 6.68 and 6.98 higher for girls .girls have greater ability of sustaining loyalty.

Love; the mean for boys and girls was found 6.44 and 6.45 . 
Care; in this virtue boys have a mean of 7.51 and girls have 8.28 , because girls are to be better relationship with home and workmates.

Wisdom ; $\mathrm{t}$ value for this virtue among boys and girls was found to be 38.33 significant at both levels again girls had a higher score of mean because they are more concerned for the mankind.

\section{Culture wise interpretations}

Hope ; The mean for virtue hope for tribals and non tribals 6.57 and 6.80 respectively, it was more for non tribals the difference was due to variation in rearing up practices of tribals and non tribals and child's relationship with mother .

Will; In this virtue the mean of tribals and non tribals were 5.25 and 5.32 respectively, was more for non tribalsthan tribals..

Purpose ; The mean among tribals and non tribals in this virtue was 5.78 and 5.79 , Competence ; the mean of this virtue of tribals and nontribals was 7.61 and 8.31 , i.e. higher for non tribals and lower for tribals tribals were higher on this virtue must have received more encouragement and commendation by parents and teachers hence they developed a feeling of competence more as compared to tribals .

Fidelity; The means of tribals and nontribals were 6.48 and 7.18 higher for non tribals .non tribals have greater ability of sustaining loyalty.

Love; The mean for tribals and nontribals was found 6.27 and 6.59 and t value 1.28 insignificant at both the levels

Care ; in this virtue tribals have a mean of 7.60 and nontribals have 8.19 , higher for nontribals.

Wisdom; .For tribals mean is 6.20 and for non tribals mean is 6.69 higher for non tribals,

\section{Conclusions;}

Profiles are matching for tribals and non tribals on virtue, will and purpose, while they differ on hope , competence, fidelity, love ,care and wisdom. Sex wise profile of boys and girls match on love and differ on hope, will. Purpose, competence, fidelity, care and wisdom, therefore counseling is needed for bringing different groups on parity.

\section{References}

[1]. Carol E Franz*, Kathleen M White (2006) Individuation and attachment in personality development: Extending Erikson's theory Journal of Personality Volume 53, Issue 2, pages 224-256.

[2]. Erik H Erikson (1993) Childhood and Society Publisher: W. W. Norton \& Company; NY, London.

[3]. Eagle, M (1997), "Contributions of Erik Erikson", Psychoanalytic review 84 (3): 337-47.

[4]. Engler, Barbara Houghton,(2006) Personality theories : An Introduction, Mifflin Company.

[5]. Erikson J. M. and Kivnick H., (1986) Vital Involvement in Old Age. . W. Norton \& Company, NY, London.

[6]. Erikson J. M., (1987) The Life Cycle Completed, W.W. Wiley Norton \& Company, NY, London. 